\title{
COMP mutation screening as an aid for the clinical diagnosis and counselling of patients with a suspected diagnosis of pseudoachondroplasia or multiple epiphyseal dysplasia
}

\author{
Jason Kennedy ${ }^{1}$, Gail Jackson ${ }^{1,2}$, Simon Ramsden ${ }^{1}$, Jacky Taylor ${ }^{1}$, William Newman ${ }^{3}$, \\ Michael J Wright ${ }^{4}$, Dian Donnai ${ }^{3}$, Rob Elles ${ }^{1}$ and Michael D Briggs ${ }^{* 2}$
}

\footnotetext{
${ }^{1}$ National Genetics Reference Laboratory (Manchester), Regional Genetics Services, St. Mary's Hospital, Manchester, UK; ${ }^{2}$ Wellcome Trust Centre for Cell-Matrix Research, Faculty of Life Sciences, University of Manchester, Manchester, UK; ${ }^{3}$ Medical Genetics, St Mary's Hospital, Manchester, UK; ${ }^{4}$ Institute of Human Genetics, International Centre for Life, Newcastle-upon-Tyne, UK
}

The skeletal dysplasias are a clinically and genetically heterogeneous group of conditions affecting the development of the osseous skeleton and fall into the category of rare genetic diseases in which the diagnosis can be difficult for the nonexpert. Two such diseases are pseudoachondroplasia (PSACH) and multiple epiphyseal dysplasia (MED), which result in varying degrees of short stature, joint pain and stiffness and often resulting in early onset osteoarthritis. PSACH and some forms of MED result from mutations in the cartilage oligomeric matrix protein (COMP) gene and to aid the clinical diagnosis and counselling of patients with a suspected diagnosis of PSACH or MED, we developed an efficient and accurate molecular diagnostic service for the COMP gene. In a 36-month period, 100 families were screened for a mutation in COMP and we identified disease-causing mutations in $78 \%$ of PSACH families and $36 \%$ of MED families. Furthermore, in several of these families, the identification of a disease-causing mutation provided information that was immediately used to direct reproductive decision-making. European Journal of Human Genetics (2005) 13, 547-555. doi:10.1038/sj.ejhg.5201374

Published online 9 March 2005

Keywords: Skeletal dysplasia; cartilage oligomeric matrix protein; gene mutation; molecular diagnosis

Introduction

The osteochondrodysplasias are a clinically and genetically heterogeneous group of conditions affecting the development of the osseous skeleton. Over 200 unique phenotypes have been described and subsequently grouped into 33 distinct diagnostic groups by the International Working

*Correspondence: Dr Michael D Briggs, Wellcome Trust Centre for Cell Matrix Research, Faculty of Life Sciences, University of Manchester, Michael Smith Building, Oxford Road, Manchester, M13 9PT, UK.

Tel: + 44161275 5642; Fax: + 44161275 5082;

E-mail: mike.briggs@man.ac.uk

Received 12 October 2004; revised 30 November 2004; accepted 8 December 2004 group on the Classification of Constitutional Disorders of Bone $^{1}$. Although individually rare, the skeletal dysplasias have an overall birth prevalence of at least 3.9 per $10000^{2-6}$ and based on a European population of 375 million, there are at least 150000 individuals in the $\mathrm{EU}$ who have a recognised bone dysplasia.

The osteochondrodysplasias fall into the category of rare genetic disease and/or condition in which the diagnosis can be difficult. The clinical diagnosis of a specific skeletal dysplasia usually involves several types of investigation such as family history, physical examination and, most importantly, a comprehensive radiographic evaluation. ${ }^{7}$ Arriving at the correct clinical diagnosis is essential because 
there are a number of complications associated with bone dysplasias. These may be, for example, orthopaedic, ${ }^{8}$ neurologic, ${ }^{8,9}$ cardiorespiratory $^{10,11}$ or psychological ${ }^{12-14}$ in nature and are often predictable and either preventable or treatable. ${ }^{8,15}$ Importantly, many complications are diagnosis specific and molecular analysis plays a valuable role not only in establishing a diagnosis and facilitating accurate risk estimation and genetic counselling but also in assisting in clinical management of the condition. ${ }^{16}$

Pseudoachondroplasia (PSACH) and multiple epiphyseal dysplasia (MED) belong to the same diagnostic group and comprise a clinical spectrum ranging from mild MED phenotypes to typical severe PSACH. ${ }^{17}$ They are characterised by various degrees of disproportionate short stature, lower limb deformities and early onset osteoarthritis. $\mathrm{PSACH}$ results exclusively from mutations in the gene encoding cartilage oligomeric matrix protein (COMP), a large pentameric glycoprotein found in cartilage tendon and ligament. The more severe forms of MED are allelic with PSACH and can also result from COMP mutations; however, MED is genetically heterogeneous and milder forms can result from mutations in the genes encoding type IX collagen and matrilin-3. ${ }^{18}$ Although diseasespecific molecular diagnostic methods for PSACH and MED have been developed, links from this basic research to applied clinical research are not well established. ${ }^{19} \mathrm{We}$ therefore established an accurate and efficient molecular diagnostic service for the COMP gene. In a 36-month period (June 2001-May 2004), 100 families were referred with a suspected diagnosis of PSACH or MED and we identified a COMP mutation in 53 of these families. In seven of these families, the identification of a diseasecausing mutation not only confirmed the clinical diagnosis of a bone dysplasia, but also provided information that has been subsequently used to direct reproductive decisionmaking and allow testing of at-risk family members.

\section{Materials and methods}

Genomic DNA was received in Manchester for our ongoing studies as part of the UK Collaborative Study of Genetic Diagnosis for Skeletal Dysplasias and the European Skeletal Dysplasia Network (www.esdn.org). In each case, a diagnosis of PSACH or MED was suspected on the clinical and radiographic presentation of the proband and other affected family members. The University of Manchester Ethical Committee approved this study and informed consent was obtained from all individuals.

PCR amplification of exons 8-19, including the immediate splice donor and acceptor sites of the COMP gene was performed using exon-specific intronic primers (Table 1) in eight separate PCR amplicons. Briefly, PCR amplifications were performed in a total volume of $40 \mu \mathrm{l}$ using a custom PCR ReddyMix ${ }^{\mathrm{TM}}$ master mix (ABgene, UK) containing
Table 1 Primer sequences for PCR amplification of exons 8-19 of the COMP gene

\begin{tabular}{|c|c|c|c|}
\hline \multicolumn{2}{|c|}{ Primer Sequence $\left(5^{\prime} \rightarrow 3^{\prime}\right)$} & $\begin{array}{c}\text { Annealing } \\
\text { temperature } \\
\left({ }^{\circ} \mathrm{C}\right)\end{array}$ & $\begin{array}{l}\text { Product } \\
\text { size } \\
(b p)\end{array}$ \\
\hline $\begin{array}{l}8 \mathrm{~F} \\
9 \mathrm{R}\end{array}$ & $\begin{array}{l}\text { TTG AGG CGG GGT TGG GTG } \\
\text { ACC GTG CCG AGC CGT AGA T }\end{array}$ & 64 & 413 \\
\hline $10 \mathrm{~F}$ & AGG AGT GTG ACC TTT GCC TTC T & 64 & 334 \\
\hline $10 \mathrm{R}$ & CTA GTC CAG CTT ACC CCA TCC & & \\
\hline $\begin{array}{l}11 \mathrm{~F} \\
12 \mathrm{R}\end{array}$ & $\begin{array}{l}\text { GAA GTC ATT CTG GCC TGG TC } \\
\text { AGC GTT TTG TCA AAG GCT ACC }\end{array}$ & 64 & 518 \\
\hline $\begin{array}{l}13 \mathrm{~F} \\
13 \mathrm{R}\end{array}$ & $\begin{array}{l}\text { CGG GTA GCC TTT GAC AAA ACG } \\
\text { GCC CGC CCA CCG TAG AC }\end{array}$ & 62 & 331 \\
\hline $14 \mathrm{~F}$ & GGC GGG CCC TGA CTT TAG & 64 & 546 \\
\hline $15 \mathrm{R}$ & ATA ACC CCG CCC CTC TGT & & \\
\hline $\begin{array}{l}16 \mathrm{~F} \\
16 \mathrm{R}\end{array}$ & $\begin{array}{l}\text { GTT CTG GGT GCC AGG TTC } \\
\text { AAG GGT TIT ACG GAG GGT CAT }\end{array}$ & 64 & 335 \\
\hline $17 \mathrm{~F}$ & TGC TCC CAA CTG TCT CTC CA & 64 & 312 \\
\hline $17 R$ & ACC TGG GCC TGT GTG TCC & & \\
\hline $18 \mathrm{~F}$ & TCT GAG AGG GAA GGG TCT GG & 64 & 443 \\
\hline $19 \mathrm{R}$ & ССС ТТС ТСА СТТ ССС ССТ СА & & \\
\hline
\end{tabular}

$0.5 \mu \mathrm{M}$ of each primer, $1.25 \mathrm{U}$ of Taq polymerase, $67 \mathrm{mM}$ Tris- $\mathrm{HCl}\left(\mathrm{pH} 8.0\right.$ at $\left.25^{\circ} \mathrm{C}\right), 16 \mathrm{mM}\left(\mathrm{NH}_{4}\right)_{2} \mathrm{SO}_{4}, 3.7 \mathrm{mM}$ $\mathrm{MgCl}_{2}, 0.085 \mathrm{mg} / \mathrm{ml} \mathrm{BSA}, 6.7 \mu \mathrm{M}$ EDTA, $0.75 \mathrm{mM}$ of each $\mathrm{dNTP}$ and 50-100 ng of genomic DNA. PCR thermal cycling consisted of an initial denaturation at $95^{\circ} \mathrm{C}$ for $3 \mathrm{~min}$, followed by 35 cycles of denaturation at $94^{\circ} \mathrm{C}$ for $30 \mathrm{~s}$, annealing $\left(62-64^{\circ} \mathrm{C}\right.$ depending on the primer annealing temperature) for $30 \mathrm{~s}$, and extension at $72^{\circ} \mathrm{C}$ for $1 \mathrm{~min}$, followed by a final extension at $72^{\circ} \mathrm{C}$ for $5 \mathrm{~min}$. PCR amplification was performed in a Dyad Disciple ${ }^{\mathrm{TM}}$ thermal cycler (MJ Research ${ }^{(}$Inc., USA). PCR products were visualised by agarose gel electrophoresis to ensure that amplification had occurred without contamination, then purified using the QuickStep ${ }^{\mathrm{TM}} 2$ PCR purification system (Edge Biosystems, USA). Purified PCR products were used as a template for bidirectional fluorescent DNA sequencing. To facilitate higher throughput mutation screening, genomic DNA dilutions at $100 \mathrm{ng} / \mu \mathrm{l}$ were aliquoted into 96-well plates. Subsequent PCR reactions were set up in 96-well PCR plates (ABgene, UK) using multi-channel pipettes (Anachem, UK). Sequencing reactions were performed with ABI PRISM ${ }^{\circledR}$ BigDye $^{\mathrm{TM}}$ Terminators v 2.0 Cycle Sequencing Ready Reaction Kit (Applied Biosystems, UK). DNA sequencing reactions were purified by gel filtration using Sephadex ${ }^{\circledR}$ G-50 (Sigma, UK) loaded Multiscreen-HV plates (Millipore, UK), then electrophoresed on either an ABI PRISM $^{\circledR} 3100$ Genetic Analyzer (Applied Biosystems, UK) or an Applied Biosystems 3730 XL DNA Analyzer (Applied Biosystems, UK). The DNA sequence data generated were compared against a reference sequence to detect variants using the Staden sequence analysis package. ${ }^{20}$ All DNA sequence traces were compared with a normal nonaffected control and checked against the published 
COMP cDNA sequence (COMP GenBank Accession Number XM_009336).

\section{Results \\ Patient referrals}

Patients with a suspected diagnosis of PSACH or MED were referred to the National Genetics Reference Laboratory (Manchester) as part of one of two studies; 'Meeting the genetic needs of families with skeletal dysplasias' or 'The European Skeletal Dysplasia Network (ESDN)'. In most cases, the referring diagnosis was made by a clinical geneticist and many of these would not have been an expert in the diagnosis of skeletal dysplasias. Furthermore, our own assessment of the available clinical and radiographic information was not a prerequisite for molecular testing.

\section{Development of an accurate and efficient COMP screening protocol}

The COMP gene is located on chromosome 19p12 and comprises 19 exons which encode the signal peptide (exon $1)$, coiled-coil (exons 1-4), type II repeats (exons 4-8), type III repeats (exons 8-15) and C-terminal domain (exons 16-19), respectively. ${ }^{21}$ All of the mutations identified to date in PSACH and MED cluster in the type III or C-terminal domains. ${ }^{18}$ We therefore established an accurate and efficient COMP mutation screening protocol by employing bidirectional fluorescent DNA sequencing of exons 8-19 of COMP.

Initially, each exon of COMP (exons 8-19) was individually amplified by PCR and sequenced as 12 separate amplicons in small panels of patients (usually 6-8 patients per panel). Oligonucleotide primers were designed to anneal at the same temperature so that different exons could be amplified by PCR in the same thermal cycler (Table 1). To improve the turn-round time two exons were amplified as a single amplimer (ie exons $8+9,11+12$, $14+15,18+19$ ); exons $8-19$ of COMP could therefore be amplified and sequenced as eight amplimers. Further improvements included the use of 96-well plates (to amplify different exons for the same panel of patients), in conjunction with multi-channel pipettes for PCR and sequencing and the use of QuickStep 2 PCR-purification system in a 96-well format, which can purify 96 PCRs in one step (to reduce pipetting error and hands-on time). During the course of this study, it was found that panels of 13 patients with the relevant controls samples could be routinely screened (ie 13 patients + one normal control+one mutant control + water control). In total, 16 samples occupied two columns of a 96-well plate and thus in one 96-well plate six PCR amplicons can be analysed for 13 patients.

In all cases, our screening approach consisted of a 'level 1 screen', which involved the analysis of exons 10 and 13, which were believed to contain the majority of COMP mutations, followed by the analysis of the remaining 10 exons if the level 1 screen proved negative. The detection of individuals who were heterozygous for point mutations was aided by the use of the Staden DNA sequence analysis package. ${ }^{20}$ Any mutations that we identified were confirmed in a fresh dilution of genomic DNA.

\section{COMP mutational analysis in suspected cases of PSACH or MED}

During the course of 3 years, affected and unaffected individuals from 100 families with a skeletal dysplasia were referred to Manchester for COMP analysis (Table 2). This cohort of patients comprised those with a suspected diagnosis of PSACH (45 affected individuals in 41 families), MED (81 affected individuals in 58 families) or SEMD (Hall type $^{22}$ (two affected individuals in one family). In this latter family (family 39), the referring clinician requested COMP analysis to rule out a possible diagnosis of PSACH. The majority of families were referred from either the UK $(n=36)$ or the rest of Europe $(n=60)$, while two cases were referred from Australia and one each from New Zealand and Singapore. Bidirectional fluorescent DNA sequencing of exons 8-19 of COMP was initially performed on the DNA of at least one affected individual from each family and when a potential mutation was identified additional family members were analysed when possible. A potential mutation was defined as an in-frame deletion or insertion or an amino-acid substitution, particularly one that affected a conserved residue within the type III or C-terminal domains of COMP. ${ }^{18}$

Sequence analysis of at least one affected individual from the 41 families with a referred diagnosis of PSACH identified disease-causing COMP mutations in 32 (78\%) of them, while analysis of the 58 families with a referred diagnosis of MED identified disease-causing COMP mutations in $21(36 \%)$ of these families (Table 2). The only previously published report documenting the frequency of COMP mutations in PSACH and MED found that $100 \%$ $(25 / 25)$ of $\mathrm{PSACH}^{23}$ and $28 \%$ (7/25) of $\mathrm{MED}^{24}$ resulted from COMP mutations. However, this cohort of patients had been preselected following a careful examination of the clinical history and relevant radiographs by the authors (Daniel Cohn, personal communication), which was often not the case with the referrals to our centre. We did not identify a COMP mutation in the single family with SEMD (Hall type) that we analysed, which confirms previous findings. ${ }^{23}$

Our inability to detect COMP mutations in $22 \%$ of the families referred with a suspected diagnosis of PSACH prompted us to reassess their clinical diagnosis. A clinical history and relevant $\mathrm{X}$-rays were obtained from seven of the nine families, and in six of these families (families $33-35,38,41$ and 42 in Table 2), a revised diagnosis was suggested, which including spondyloepiphyseal dysplasia 


\begin{tabular}{|c|c|c|c|c|c|c|c|c|c|c|c|}
\hline Family & \multicolumn{3}{|c|}{$\begin{array}{l}\text { No. of family } \\
\text { members }\end{array}$} & $\begin{array}{l}\text { Country } \\
\text { of } \\
\text { referral }\end{array}$ & $\begin{array}{c}\text { Diagnosis } \\
\text { on } \\
\text { referral }\end{array}$ & Inheritance & Exon & $\begin{array}{l}\text { COMP } \\
\text { mutation } \\
\text { (nucleotide) }\end{array}$ & $\begin{array}{l}\text { COMP defect } \\
\text { (predicted } \\
\text { amino-acid } \\
\text { change) }\end{array}$ & $\begin{array}{l}\text { Previously published } \\
\text { or novel mutation }^{a}\end{array}$ & $\begin{array}{l}\text { Differential } \\
\text { diagnosis } \\
\text { and/or other } \\
\text { gene mutation }\end{array}$ \\
\hline 1 & 2 & 1 & 0 & UK & PSACH & $A D^{b}$ & 10 & c. $1052 \mathrm{G}>\mathrm{A}$ & p.Cys351Tyr & $\begin{array}{l}\text { Mabuchi et al } \\
(2003)^{26}\end{array}$ & \\
\hline 2 & 1 & 0 & 0 & $\mathrm{~F}$ & PSACH & Sporadic? & 10 & c. $1127 \mathrm{~A}>\mathrm{T}$ & p.Asp376Val & Novel & \\
\hline 3 & 1 & 0 & 0 & UK & PSACH & $\mathrm{n} / \mathrm{d}$ & 13 & c. $1417 \mathrm{G}>\mathrm{A}$ & p.Asp473Asn & Deere et al $(1998)^{27}$ & \\
\hline 4 & 1 & 0 & 0 & UK & PSACH & $n / d$ & 13 & c. $1318 \mathrm{G}>\mathrm{A}$ & p.Gly440Arg & $\begin{array}{l}\text { Loughlin et al } \\
(1998)^{28}\end{array}$ & \\
\hline 5 & 1 & 0 & 1 & UK & PSACH & $A D^{b}$ & 13 & c.1417_1419 delGAC & p.Asp473 del & Hecht et al (1995) & \\
\hline 6 & 1 & 0 & 0 & $\mathrm{CH}$ & PSACH & $\mathrm{n} / \mathrm{d}$ & 13 & c. $1318 \mathrm{G}>\mathrm{A}$ & p.Gly440Arg & $\begin{array}{l}\text { Loughlin et al } \\
(1998)^{28}\end{array}$ & \\
\hline 7 & 1 & 0 & 0 & $\mathrm{CH}$ & PSACH & $\mathrm{n} / \mathrm{d}$ & 13 & c.1417_1419 delGAC & p.Asp473 del & Hecht et al (1995) & \\
\hline 8 & 1 & 0 & 0 & UK & PSACH & $n / d$ & 11 & c.1170_1181del insTGT & $\begin{array}{l}\text { p.Pro391_Asp394del } \\
\text { insVal }\end{array}$ & $\begin{array}{l}\text { Loughlin et al } \\
(1998)^{28}\end{array}$ & \\
\hline 9 & 1 & 0 & 1 & $\mathrm{TR}$ & PSACH & $A D$ & 9 & c. $925 \mathrm{G}>\mathrm{A}$ & p.Gly309Arg & Delot et al $(1998)^{30}$ & \\
\hline 10 & 1 & 0 & 0 & UK & PSACH & Sporadic & 10 & c.1021_1026 del & p.Glu341_Asp342 del & I Novel & \\
\hline 11 & 1 & 2 & 0 & UK & PSACH & Sporadic & 14 & c. $1552 \mathrm{G}>\mathrm{A}$ & p.Asp518Asn & Ikegawa et al (1998) & \\
\hline 12 & 1 & 3 & 0 & A & PSACH & Sporadic & 14 & c. $1528 \_1539 \mathrm{del}$ & p.Ala510_513Val del & Novel & \\
\hline 13 & 1 & 2 & 0 & $\mathrm{E}$ & PSACH & Sporadic $^{c}$ & 13 & c. 1393 G > C & p.Gly465Ärg & Novel & \\
\hline 14 & 1 & 0 & 0 & $\mathrm{D}$ & PSACH & $n / d$ & 13 & c. $1417 \_1419$ delGAC & p.Asp473 del & Hecht et al (1995) & \\
\hline 15 & 1 & 0 & 0 & $\mathrm{D}$ & PSACH & Sporadic ${ }^{c}$ & 8 & c.831_851 del & p.Asp277_Pro283 del & Novel & \\
\hline 16 & 2 & 1 & 0 & NZ & PSACH & $A D^{b}$ & 18 & c. $215 \overline{5} \mathrm{G}>\mathrm{A}$ & p.Gly719Ser & Novel & \\
\hline 17 & 1 & 0 & 1 & UK & PSACH & $A D$ & 10 & c. $1114 \_1116$ del & p.Asp372 del & Briggs et al (1995) & \\
\hline 18 & 1 & 0 & 1 & UK & PSACH & $A D$ & 13 & c.1417 1419 delGAC & p.Asp473 del & Hecht et al (1995) & \\
\hline 19 & 1 & 0 & 0 & UK & PSACH & $\mathrm{n} / \mathrm{d}$ & 9 & c. $893 \bar{C}>T$ & p.Ser298Leu & Novel & \\
\hline 20 & 2 & 0 & 0 & UK & PSACH & $A D$ & 14 & c. $1526 \mathrm{~A}>\mathrm{G}$ & p.Asp509Gly & Deere et al (1998) & \\
\hline 21 & 1 & 0 & 0 & $\mathrm{D}$ & PSACH & $n / d$ & 13 & c.1417_1419 delGAC & Asp473 del & Hecht et al (1995) & \\
\hline 22 & 1 & 0 & 0 & UK & PSACH & $A D$ & 13 & c. $1412 A>G$ & p.Asp471Gly & Novel & \\
\hline 23 & 1 & 0 & 0 & $\mathrm{NL}$ & PSACH & $\mathrm{n} / \mathrm{d}$ & 13 & c.1417_1419 delGAC & p.Asp473 del & Hecht et al (1995) ${ }^{29}$ & \\
\hline 24 & 1 & 0 & 0 & UK & PSACH & $\mathrm{n} / \mathrm{d}$ & 13 & c.1417_1419 delGAC & p.Asp473 del & Hecht et al (1995) 29 & \\
\hline 25 & 1 & 2 & 1 & 1 & PSACH & $\mathrm{n} / \mathrm{d}$ & 10 & c. $1021 \_1026 \mathrm{del}$ & p.Glu341_Asp342 del & I Novel & \\
\hline 26 & 1 & 2 & 0 & & Singapore & PSACH & Sporadic & & c.1417_1419 delGAC & p.Asp473 del & Hecht et al (1995) $)^{29}$ \\
\hline 27 & 1 & 0 & 0 & 1 & PSACH & $\mathrm{n} / \mathrm{d}$ & 13 & c. $1423 \mathrm{G}>\mathrm{A}$ & p.Asp475Asn & Deere et al (1998) & \\
\hline 28 & 1 & 0 & 0 & G & PSACH & $\mathrm{n} / \mathrm{d}$ & 13 & c.1417_1419 delGAC & p.Asp473 del & Hecht et al (1995) & \\
\hline 29 & 1 & 0 & 0 & $\mathrm{~F}$ & PSACH & $\mathrm{n} / \mathrm{d}$ & 13 & c. $1435 \mathrm{G}>\mathrm{T}$ & p.Asp479Tyr & Novel & \\
\hline 30 & 1 & 0 & 0 & $\mathrm{~F}$ & PSACH & $n / d$ & 14 & c. $1511 \mathrm{G}>\mathrm{C}$ & p.Cys504Ser & Novel & \\
\hline 31 & 1 & 0 & 0 & TR & PSACH & $n / d$ & 14 & c. $1586 \mathrm{C}>\mathrm{T}$ & p.Thr529lle & Novel & \\
\hline 32 & 1 & 0 & 0 & $\mathrm{D}$ & PSACH & $\mathrm{n} / \mathrm{d}$ & 14 & c. $1553 A>G$ & p.Asp518Gly & Novel & \\
\hline 33 & 1 & 1 & 0 & B & PSACH & $n / d$ & & & No & & SEMD unclassified \\
\hline 34 & 1 & 0 & 0 & UK & PSACH & $n / d$ & & & No & & SEDc/Kniest \\
\hline 35 & 2 & 2 & 0 & G & PSACH & $\begin{array}{l}\text { AR or } \\
\text { mosaicism }\end{array}$ & & & No & & SEMD unclassified \\
\hline 36 & 1 & 0 & 0 & UK & $\mathrm{PSACH} / \mathrm{SML}$ & $\mathrm{Dn} / \mathrm{d}$ & & & No & & SMD unclassified \\
\hline 37 & 1 & 0 & 0 & $G$ & PSACH & $A D$ & & & No & & $\mathrm{n} / \mathrm{d}$ \\
\hline 38 & 1 & 0 & 0 & UK & PSACH & $\mathrm{n} / \mathrm{d}$ & & & No & & Brachy C \\
\hline 39 & 2 & 2 & 0 & UK & SEMD & $A D$ & & & No & & SEMD (Hall type $)^{22}$ \\
\hline 40 & 1 & 0 & 0 & UK & PSACH & $\mathrm{n} / \mathrm{d}$ & & & No & & $\mathrm{n} / \mathrm{d}$ \\
\hline
\end{tabular}

Table 2 Summary of COMP analysis in 100 patients with PSACH, MED or SEMD 
Table 2 (Continued)

\begin{tabular}{|c|c|c|c|c|c|c|c|c|c|c|c|}
\hline \multirow[b]{2}{*}{ Family } & \multicolumn{3}{|c|}{$\begin{array}{l}\text { No. of family } \\
\text { members }\end{array}$} & \multirow[t]{2}{*}{$\begin{array}{l}\text { Country } \\
\text { of } \\
\text { referral }\end{array}$} & \multirow[t]{2}{*}{$\begin{array}{c}\text { Diagnosis } \\
\text { on } \\
\text { referral }\end{array}$} & \multirow{2}{*}{ Inheritance } & \multirow[t]{2}{*}{ Exon } & \multirow[t]{2}{*}{$\begin{array}{l}\text { COMP } \\
\text { mutation } \\
\text { (nucleotide) }\end{array}$} & \multirow[t]{2}{*}{$\begin{array}{l}\text { COMP defect } \\
\text { (predicted } \\
\text { amino-acid } \\
\text { change) }\end{array}$} & \multirow[t]{2}{*}{$\begin{array}{l}\text { Previously published } \\
\text { or novel mutation }\end{array}$} & \multirow[t]{2}{*}{$\begin{array}{l}\text { Differential } \\
\text { diagnosis } \\
\text { and/or other } \\
\text { gene mutation }\end{array}$} \\
\hline & A & $\mathrm{N}$ & $\mathrm{U}$ & & & & & & & & \\
\hline$\overline{41}$ & 1 & 0 & 0 & UK & PSACH & $\mathrm{n} / \mathrm{d}$ & & & No & & $\mathrm{CHH}$ \\
\hline 42 & 1 & 0 & 0 & $\mathrm{~F}$ & PSACH & $n / d$ & & & No & & SEMD unclassified \\
\hline 43 & 1 & 0 & 0 & D & MED & Sporadic & 10 & c. $1096 \mathrm{G}>\mathrm{C}$ & p.Gly366Arg & Novel & \\
\hline 44 & 2 & 2 & 0 & UK & MED & $A D^{b}$ & 16 & c. $1813 \mathrm{G}>\mathrm{C}$ & p.Asp605Asn & Novel & \\
\hline 45 & 1 & 4 & 0 & UK & MED & Sporadic ${ }^{c}$ & 11 & c. $1220 \mathrm{G}>\mathrm{T}$ & p.Cys407Phe & Novel & \\
\hline 46 & 2 & 1 & 0 & $\mathrm{D}$ & MED & $A D^{b}$ & 10 & c. $1132 \mathrm{G}>\mathrm{T}$ & p.Asp378Tyr & Novel & \\
\hline 47 & 2 & 0 & 0 & UK & MED & $A D^{b}$ & 8 & c. $827 \mathrm{C}>\mathrm{G}$ & p.Pro276Arg & $\begin{array}{l}\text { Czarny-Ratajczak et al } \\
(2001)^{32}\end{array}$ & \\
\hline 48 & 4 & 1 & 0 & UK & MED & $A D^{b}$ & 14 & c. $1665 \mathrm{C}>\mathrm{G}$ & p.Asn555Lys & Novel & \\
\hline 49 & 3 & $i$ & 0 & 1 & MED & $A D^{b}$ & 11 & c. $1154 \_1156$ del & p.Asn386 del & Novel & \\
\hline 50 & 1 & 2 & 0 & UK & MED & $A D^{b}$ & 13 & c. $1475 \mathrm{~A}>\mathrm{C}$ & p.GIn492Pro & Novel & \\
\hline 51 & 1 & 2 & 0 & D & MED & Sporadic $^{c}$ & 13 & c. $1315 \mathrm{G}>\mathrm{A}$ & p.Asp439Asn & Novel & \\
\hline 52 & 2 & $\overline{3}$ & 0 & UK & MED & $A D^{b, c}$ & 11 & c. $1195 \mathrm{G}>\mathrm{A}$ & p.Asp399Asn & Novel & \\
\hline 53 & 5 & 3 & 0 & UK & MED & $A D^{b}$ & 11 & c. $1153 \mathrm{G}>\mathrm{A}$ & p.Asp385Asn & Mabuchi et al & \\
\hline 54 & 1 & 2 & 0 & UK & MED & $A D^{b}$ & 9 & c.929 A > T & p.Asp310Val & $\begin{array}{l}(2003)^{2} \\
\text { Novel }\end{array}$ & \\
\hline 55 & 1 & 2 & 0 & UK & MED & Sporadic ${ }^{c}$ & 10 & C. $1102 \mathrm{G}>\mathrm{C}$ & p.Gly368Arg & Novel & \\
\hline 56 & 2 & 0 & 0 & UK & MED & $A D^{b}$ & 14 & c. $1665 \mathrm{C}>\mathrm{A}$ & p.Asn555Lys & Novel & \\
\hline 57 & 4 & 2 & 0 & UK & MED & $A D^{b}$ & 11 & c. $1153 \mathrm{G}>\mathrm{A}$ & p.Asp385Asn & Mabuchi et al & \\
\hline 58 & 1 & 0 & 0 & UK & MED & $\mathrm{n} / \mathrm{d}$ & 11 & c. $1153 \mathrm{G}>\mathrm{A}$ & p.Asp385Asn & $\begin{array}{l}\text { Mabuchi et al } \\
(2003)^{26}\end{array}$ & \\
\hline 59 & 1 & 0 & 0 & $\mathrm{D}$ & MED & $\mathrm{n} / \mathrm{d}$ & 18 & c. $2153 \mathrm{G}>\mathrm{C}$ & p.Arg718Pro & Novel & \\
\hline 60 & 1 & 0 & 0 & UK & MED & $\mathrm{n} / \mathrm{d}$ & 13 & c. $1435 \mathrm{G}>\mathrm{C}$ & pAsp479His & Novel & \\
\hline 61 & 1 & 0 & 0 & UK & MED & $\mathrm{n} / \mathrm{d}$ & 13 & c. $1362 \mathrm{~T}>\mathrm{G}$ & p.Ser454Arg & Novel & \\
\hline 62 & 1 & 1 & 0 & $\mathrm{CH}$ & MED & $\mathrm{n} / \mathrm{d}$ & 11 & c. $1153 \mathrm{G}>\mathrm{A}$ & p.Asp385Asn & $\underset{(2003)^{26}}{\text { Mabuchi et al }}$ & \\
\hline 63 & 1 & 0 & 0 & NL & MED & $\mathrm{n} / \mathrm{d}$ & 11 & c. $1201 \mathrm{G}>\mathrm{A}$ & p.Asp401Asn & Novel & \\
\hline 64 & 1 & 0 & 0 & $\mathrm{D}$ & MED & Sporadic & & & No & & DTDST \\
\hline 65 & 2 & 1 & 0 & UK & MED & $A D$ & & & No & & $\mathrm{E} 134 \mathrm{~K}$ in $M A T N 3^{33}$ \\
\hline 66 & 3 & 1 & 0 & UK & MED & $A D$ & & & No & & $\mathrm{A} 219 \mathrm{D}$ in $M A T N 3^{33}$ \\
\hline 67 & 1 & 0 & 0 & $\mathrm{~B}$ & MED & $\begin{array}{l}\text { AR/ } \\
\text { Mosaicism }\end{array}$ & & & No & & $\mathrm{R} 121 \mathrm{~W}$ in MATN $3^{33}$ \\
\hline 68 & 1 & 0 & 0 & UK & MED & Sporadic & & & No & & Synovial \\
\hline 69 & 1 & 0 & 0 & UK & MED & $\mathrm{n} / \mathrm{d}$ & & & No & & ASPED \\
\hline 70 & 1 & 0 & 0 & UK & MED & $\mathrm{n} / \mathrm{d}$ & & & No & & \\
\hline 71 & 1 & 0 & 0 & $\mathrm{D}$ & MED & $n / d$ & & & No & & \\
\hline 72 & 1 & 0 & 0 & Aust & MED & Sporadic & & & No & & \\
\hline 73 & 1 & 0 & 0 & UK & MED & $n / d$ & & & No & & \\
\hline 74 & 1 & 2 & 0 & B & MED & $\mathrm{n} / \mathrm{d}$ & & & No & & \\
\hline 75 & 1 & 0 & 0 & UK & MED & $\mathrm{n} / \mathrm{d}$ & & & No & & \\
\hline 76 & 1 & 0 & 0 & UK & MED & $\mathrm{AD} ?$ & & & No & & \\
\hline 77 & 1 & 0 & 0 & UK & MED & Sporadic & & & No & & \\
\hline 78 & 1 & 0 & 0 & UK & MED & $n / d$ & & & No & & \\
\hline 79 & 1 & 0 & 0 & UK & MED & $\mathrm{n} / \mathrm{d}$ & & & No & & \\
\hline 80 & 2 & 1 & 1 & UK & MED & $A D$ & & & No & & \\
\hline
\end{tabular}


Table 2 (Continued)

\begin{tabular}{|c|c|c|c|c|c|c|c|c|c|c|c|}
\hline \multirow[b]{2}{*}{ Family } & \multicolumn{3}{|c|}{$\begin{array}{l}\text { No. of family } \\
\text { members }\end{array}$} & \multirow[t]{2}{*}{$\begin{array}{c}\text { Country } \\
\text { of } \\
\text { referral }\end{array}$} & \multirow[t]{2}{*}{$\begin{array}{l}\text { Diagnosis } \\
\text { on } \\
\text { referral }\end{array}$} & \multirow[t]{2}{*}{ Inheritance } & \multirow[t]{2}{*}{ Exon } & \multirow[t]{2}{*}{$\begin{array}{l}\text { COMP } \\
\text { mutation } \\
\text { (nucleotide) }\end{array}$} & \multirow[t]{2}{*}{$\begin{array}{l}\text { COMP defect } \\
\text { (predicted } \\
\text { amino-acid } \\
\text { change) }\end{array}$} & \multirow[t]{2}{*}{$\begin{array}{l}\text { Previously published } \\
\text { or novel mutation }\end{array}$} & \multirow[t]{2}{*}{$\begin{array}{l}\text { Differential } \\
\text { diagnosis } \\
\text { and/or other } \\
\text { gene mutation }\end{array}$} \\
\hline & A & $\mathrm{N}$ & U & & & & & & & & \\
\hline 81 & 1 & 0 & 0 & UK & MED & Sporadic & & & No & & \\
\hline 82 & 1 & 0 & 0 & UK & MED & $\mathrm{n} / \mathrm{d}$ & & & No & & \\
\hline 83 & 1 & 0 & 0 & UK & MED & $\mathrm{n} / \mathrm{d}$ & & & No & & \\
\hline 84 & 1 & 2 & 0 & UK & MED & Sporadic & & & No & & \\
\hline 85 & 1 & 0 & 0 & UK & MED & $\mathrm{n} / \mathrm{d}$ & & & No & & \\
\hline 87 & 1 & 0 & 0 & UK & MED & $\mathrm{n} / \mathrm{d}$ & & & No & & \\
\hline 88 & 1 & 0 & 0 & $\mathrm{D}$ & MED & $n / d$ & & & No & & \\
\hline 89 & 1 & 0 & 0 & UK & MED & $\mathrm{n} / \mathrm{d}$ & & & No & & \\
\hline 90 & 1 & 0 & 0 & D & MED & $\mathrm{n} / \mathrm{d}$ & & & No & & \\
\hline 91 & 1 & 0 & 0 & Aust & MED & AD? & & & No & & \\
\hline 92 & 1 & 0 & 0 & D & MED & $n / d$ & & & No & & \\
\hline 93 & 2 & 0 & 0 & B & MED & $\mathrm{n} / \mathrm{d}$ & & & No & & \\
\hline 94 & 1 & 0 & 0 & D & MED & Sporadic & & & No & & \\
\hline 95 & 1 & 0 & 0 & UK & MED & $\mathrm{n} / \mathrm{d}$ & & & No & & \\
\hline 96 & 2 & 0 & 0 & UK & MED & $A D^{c}$ & & & No & & \\
\hline 99 & 1 & 0 & 0 & UK & MED & $\mathrm{n} / \mathrm{d}$ & & & No & & \\
\hline 100 & 1 & 0 & 0 & UK & MED & $\mathrm{n} / \mathrm{d}$ & & & No & & \\
\hline
\end{tabular}

A: affected; N: not affected; U: unknown.

UK: United Kingdom; B: Belgium; D: Germany; F: France; NL: Netherlands; CH: Switzerland; TR: Turkey; E: Spain; I: Italy; Aust: Australia.

AD: autosomal dominant; AR: autosomal recessive.

${ }^{\text {aD }}$ Data from Human Gene Mutation Database (HGMD) ${ }^{25}$

${ }^{b}$ Affected parent and/or other family members carry mutation while unaffected and/or other family members do not have mutation.

'Unaffected parents and/or other family members do not carry mutation.

n/d: Not determined. ASPED: Trichorhinophalangeal dysplasia and angel-shaped phalangoepiphyseal dysplasia; Brachy C: Brachy-dactyly type C; SEMD-JL: Spondylo-epi-metaphyseal dysplasia with joint laxity, Hall type. Nomenclature in accordance with the current Human Genome Variation Society guidelines (http://www/hgvs.org). 
congenita (SEDc) and unclassified forms of skeletal dysplasia with radiographic characteristics of spondyloepimetaphyseal dysplasia (SEMD) or spondylometaphyseal dysplasia (SMD). In family 37, a diagnosis of PSACH was thought unlikely but a differential diagnosis could not be suggested, while in family 36 a diagnosis of an unclassified SMD had been suggested on referral, but we were unable to examine the clinical history and radiographs ourselves. This was also the case with the proband in family 40 who was noted to be lacking some features usually associated with PSACH (such as joint laxity), but we were unable to follow this up. Thus, this retrospective analysis of the available clinical and radiographic indicators highlights the difficulty in diagnosing PSACH, the clinical and radiographic overlap that exists between PSACH and various unclassified forms of SEMD and SMD and supports a COMP mutation frequency of nearly $100 \%(31 / 32)$ in clinically confirmed cases of PSACH.

Although our detection rate of COMP mutations in MED was higher than previously reported $(36 \%$ compared to $28 \%$ ), we, nonetheless, reassessed the clinical diagnosis when possible (only two families in total) and a revised diagnosis was suggested in both cases (families 68 and 69). Furthermore, in four families mutations were identified in the other genes known to cause MED such as DTDST (patient 64) and MATN3 (patients 65-67).

In addition to the disease-causing mutations, we also identified three single nucleotide polymorphisms (SNPs); these being synonymous (type III SNP), nonsynonymous (type I SNP) and noncoding (type V SNP), respectively (Table 3). The relatively few polymorphisms in COMP contrasts with the other genes involved in the skeletal dysplasias, such an SLC26A2 (DTDST) and the collagen types II, IX and XI genes, which are all highly polymorphic. The paucity of polymorphisms in COMP therefore makes it highly likely that any nonsynonymous change identified is likely to be disease-causing, however, care should still be taken when determining if a DNA change is a disease-causing mutation. For example, it was surprising that the type I SNP (N386D) was not diseasecausing because it affects a highly conserved residue and mutations at neighbouring residues (D385N and C387G) have been shown to cause MED and PSACH, respectively.

The identification of a causative mutation in families with PSACH or MED not only confirmed their clinical
Table 3 SNPs identified in COMP

\begin{tabular}{|c|c|c|c|c|}
\hline $\begin{array}{l}\text { SNP } \\
\text { Nucleotide change }\end{array}$ & Protein change & SNP type & Exon & $\begin{array}{c}\text { Allele } \\
\text { frequency }\end{array}$ \\
\hline c. $1156 A>G$ & Asn386Asp & I & 11 & 0.03 \\
\hline c. $1755 \mathrm{G}>\mathrm{A}$ & Thr585Thr & III & 16 & 0.05 \\
\hline c. $2289 \mathrm{~A}>\mathrm{G}$ & No change & V & $3^{\prime}$ UTR & 0.005 \\
\hline
\end{tabular}

diagnosis but in seven families provided information that was immediately useful for reproductive decision-making and genetic testing of at-risk family members (Table 4). These follow-on referrals included presymptomatic and prenatal diagnosis, while one family intended to use the information in conjunction with in vitro fertilisation for preimplantation diagnosis.

\section{Discussion}

The correct diagnosis of many skeletal dysplasias is a timeconsuming and complicated process involving the clinical and radiographic evaluation of the proband and other family members. ${ }^{7}$ In many cases, the clinical and radiographic findings are used to determine a differential diagnosis prior to a confirmatory molecular test. To improve this procedure, we have established an accurate and efficient molecular diagnostic protocol for COMP gene analysis of patients with PSACH or MED. Over a 3-year period, we screened the COMP gene in 100 families with a suspected diagnosis of PSACH or MED and identified disease-causing COMP mutations in over half of them (53\%). Of the 53 COMP mutations identified, 23 (43\%) had previously been published (data from Human Gene Mutation Database), ${ }^{25}$ whereas 30 mutations (57\%) were novel to a single family ( $44 \%$ of PSACH and $76 \%$ of MED families, respectively).

We identified the recurrent PSACH mutation (p.Asp473 del, often referred to as $\Delta \mathrm{D} 469$, in exon 13$)$ in $9 / 32(28 \%)$ mutations, which agrees with the previously published frequency, ${ }^{18}$ while in-frame deletions/insertions on the whole represented 15/32 (47\%) of the mutations identified in PSACH, the remaining 53\% of mutations being missense

Table 4 Type of re-referral and outcome if known

\begin{tabular}{|c|c|c|c|}
\hline Family & Diagnosis & Request & Outcome \\
\hline 13 & PSACH & Preimplantation genetic diagnosis & Unknown \\
\hline 18 & PSACH & Suspected diagnosis of PSACH in 14-year-old sister of the proband & Negative for mutation \\
\hline 5 & PSACH & Prenatal diagnosis & Negative for mutation \\
\hline 9 & PSACH & Predictive testing in the infant son of the proband & Positive for mutation \\
\hline 17 & PSACH & Predictive testing in the 8 -month-old son of the proband & Positive for mutation \\
\hline 25 & PSACH & Predictive testing in the 9 -month-old son of the proband & Negative for mutation \\
\hline
\end{tabular}




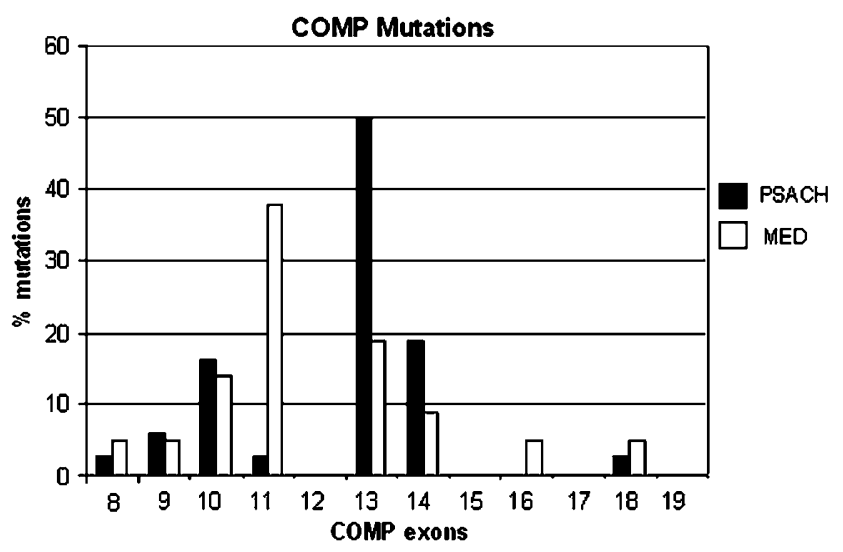

Figure 1 Genomic distribution of 53 COMP mutations identified patients with pseudoachondroplasia or multiple epiphyseal dysplasia. Note: exon 13 contains the recurrent PSACH mutation p.Asp473 del (often referred to as $\Delta \mathrm{D} 469$ ).

mutations. In contrast, only one in-frame deletion (5\%) was identified in a patient referred with MED; the remaining mutations were all missense mutations. Furthermore the genomic distribution of mutations also differed between PSACH and MED (Figure 1). While the majority of PSACH mutations were located in exon 13 (50\%), only $19 \%$ of MED mutations were found in exon 13, whereas the majority (38\%) of MED mutations were found in exon 11. This distribution is clearly a reflection of the predominance of in-frame deletions found in PSACH patients. Interestingly, our structured screening strategy meant that a 'level 1 screen' (exons 10 and 13) would on average detect $66 \%$ of the COMP mutations found in PSACH patients and $33 \%$ of the COMP mutations found in MED patients. Therefore, by modifying our level 1 screen and targeting exons 10, 13 and 14 in PSACH and 10, 11 and 13 in MED, we would in future be able to identify 85 and $71 \%$ of COMP mutations in PSACH and MED, respectively.

Our higher than expected detection of COMP mutations in MED (36\% compared with 28\%) may in part be the result of our sensitive screening protocol, which included the use of a DNA sequence analysis package (ie Staden). Alternatively, our referrals were primarily from clinical genetic departments and as such these cases may be representative of the more severe forms of MED and therefore more likely to be caused by COMP mutations.

The fact that six families had already proceeded to use their genetic test result to inform reproductive decisionmaking or facilitate testing for at-risk family members is interesting and shows the clinical utility of genetic testing for these conditions (Table 4). In three of these families (families 9, 17 and 25) predictive testing was performed on infants under the age of 9 months, when it is difficult for the nonexpert to detect the radiographic changes that are indicative of PSACH. In all six families, the diagnosis was PSACH, which probably reflects an increased desire to use genetic information to direct future decisions in this clinically more severe condition, rather than an increased detection of mutations in the PSACH patient group.

Previous publications have suggested that PSACH results exclusively from COMP mutations; ${ }^{18,23}$ however in our cohort of PSACH patients we were only able to identify mutations in $78 \%$ of them, which highlights the difficulty in arriving at a differential diagnosis and the important role that molecular diagnosis can play in the diagnostic process. Re-evaluation of the clinical and radiographic indicators suggested a revised diagnosis in most of these cases and indeed mutations were subsequently found in other genes in two of those cases. This successful outcome highlights the need for an integrated diagnostic network for rare diseases when the diagnosis is difficult for the nonexpert. Accurate diagnosis in the skeletal dysplasias and the most cost-effective use of mutation analysis requires a multidisciplinary approach, in particular the integration of clinical diagnostic and radiographic skills with the clinical molecular genetics laboratory. The disparate nature of these conditions necessitates an accurate assessment of the available clinical information to target a molecular analysis to the most likely causative gene. The identification of a disease-causing mutation will then provide essential qualitative information for diagnostic, reproductive and genetic counselling purposes. In addition, the development of a clinical genetic testing service for COMP mutation analysis may provide a paradigm for the development of mutation screening services for complex genes. The creation of a screening strategy; refinement of analysis, for example, by screening exon pairs in single amplimers; and the close liaison between clinicians and laboratory scientists to maximise efficiency of mutation detection are consistent with the objectives set out in the recent UK Government White Paper (Our inheritance our future) ${ }^{34}$ for the screening of complex genes.

\section{Acknowledgements}

We sincerely thank our colleagues from the European Skeletal Dysplasia Network ( www.ESDN.org) for their help with this project; Valerie Cormier-Daire, Christine Hall, Martine Le Merrer, Geert Mortier, Jurgen Spranger, Andrea Superti-Furga, Sheila Unger, Bernhard Zabel and Andreas Zankl. In addition, we thank David Crauford and Saima Alam for help in recruiting some of the patients used in this study. This work was supported by Health Foundation (formerly the PPP Health Care Medical Trust) and the European Commission (Project Contract Number QLG1-CT-2001-02188). MDB is Wellcome Trust Senior Research Fellow.

\section{References}

1 Hall CM: International nosology and classification of constitutional disorders of bone (2001). Am J Med Genet 2002; 113: 65-77.

2 Gustavson KH, Jorulf H: Different types of osteochondrodysplasia in a consecutive series of newborns. Helv Paediatr Acta 1975; 30: $307-314$. 
3 Camera G, Mastroiacovo P: Birth prevalence of skeletal dysplasias in the Italian Multicentric Monitoring System for Birth Defects; in: Papadatos CJ, Bartsocas CS (eds): Skeletal dysplasias. New York: Alan R. Liss, 1982, pp 441-449.

4 Orioli IM, Castilla EE, Barbosa-Neto JG: The birth prevalence rates for the skeletal dysplasias. J Med Genet 1986; 23: $328-332$.

5 Andersen Jr PE: Prevalence of lethal osteochondrodysplasias in Denmark [see comments]. Am J Med Genet 1989; 32: 484-489.

6 Stoll C, Dott B, Roth M-P, Alembik Y: Birth prevalence of skeletal dysplasias. Clin Genet 1989; 35: 88-92.

7 Mortier GR: The diagnosis of skeletal dysplasias: a multidisciplinary approach. Eur J Radiol 2001; 40: 161-167.

8 Bassett GS: The osteochondrodyslpasias; in: Morrissy R, Weinstein SL (eds): Pediatric orthopedics. Philadelphia, PA: LippincottRaven, 1996, vol 1, pp 203-254.

9 Pauli RM, Horton UK, Glinski LP, Reiser CA: Prospective assessment of risks for cervico-medullary compression in infants with achondroplasia. Am J Hum Genet 1995; 56: 732-744.

10 Tasker RC, Dundas I, Laverty A, Fletcher M, Lane R, Stocks J: Distinct patterns of respiratory difficulty in young children with achondroplasia: a clinical, sleep and lung function study. Arch Dis Child 1998; 79: 99-108.

11 Mogayzel Jr PJ, Caroll JL, Loughlin GM, Hurko O, Francomano CA, Marcus CL: Sleep-disordered breathing in children with achondroplasia. J Paediatr 1998; 132: 667-671.

12 Hill V, Sahhar M, Aitken M, Savarirayan R, Metcalfe S: Experiences at the time of diagnosis of parents who have a child with a bone dysplasia resulting in short stature. Am J Med Genet 2003; 122A: 100-107.

13 Hunter AG: Some psycholgical aspects of nonlethal chondrodysplasias: II. Depression and anxiety. Am J Med Genet 1998; 78: 9-12.

14 Hunter AG, Bankier A, Rogers JG, Sillence D, Scott Jr CI: Medical complications of achondroplasia: a multicentre patient review. J Med Genet 1998; 35: 705-712.

15 Aryanpur J, Hurko O, Francomano C et al: Craniocervical decompression in pediatric patients with achondroplasia. J Neurosurg 1990; 73: 375.

16 Unger S: A genetic approach to the diagnosis of skeletal dysplasia. Clin Orthop 2002; 32-38.

17 Rimoin DL, Rasmussen IM, Briggs MD et al: A large family with features of pseudoachondroplasia and multiple epiphyseal dysplasia: exclusion of seven candidate gene loci that encode proteins of the cartilage extracellular matrix. Hum Genet 1994; 93: $236-242$.

18 Briggs MD, Chapman KL: Pseudoachondroplasia and multiple epiphyseal dysplasia: mutation review, molecular interactions, and genotype to phenotype correlations. Hum Mutat 2002; 19: $465-478$
19 Newman B, Donnah D, Briggs MD: Molecular diagnosis is important to confirm suspected pseudoachondroplasia. $J$ Med Genet 2000; 37: 64-65, (letter).

20 Bonfield JK, Rada C, Staden R: Automated detection of point mutations using fluorescent sequence trace subtraction. Nucleic Acids Res 1998; 26: 3404-3409.

21 Newton G, Weremowicz S, Morton CC et al: Characterization of human and mouse cartilage oligomeric matrix protein. Genomics 1994; 24: 435-439.

22 Hall CM, Elcioglu NH, Shaw DG: A distinct form of spondyloepimetaphyseal dysplasia with multiple dislocations. J Med Genet 1998; 35: 566-572.

23 Cohn DH, King LM, Unger SL: Locus homogeneity in pseudoachondroplasia. Am J Hum Genet 2000; 67: 369.

24 Unger SL, King LM, Sobetzko D, Superti-Furga A, Cohn DH: A multiplcity of loci for multiple epiphyseal dysplasia. Am J Hum Genet 2000; 67: 371.

25 Stenson PD, Ball EV, Mort $\mathrm{M}$ et al: Human Gene Mutation Database (HGMD): 2003 update. Hum Mutat 2003; 21: 577-581.

26 Mabuchi A, Manabe N, Haga N et al: Novel types of COMP mutations and genotype-phenotype association in pseudoachondroplasia and multiple epiphyseal dysplasia. Hum Genet 2003; 112: 84-90.

27 Deere M, Sanford T, Ferguson HL, Daniels K, Hecht JT: Identification of twelve mutations in cartilage oligomeric matrix protein (COMP) in patients with pseudoachondroplasia. Am Med Genet 1998; 80: 510-513.

28 Loughlin J, Irven C, Mustafa Z et al: Identification of five novel mutations in cartilage oligomeric matrix protein gene in pseudoachondroplasia and multiple epiphyseal dysplasia [In Process Citation]. Hum Mutat 1998; Suppl 1: S10-S17.

29 Hecht JT, Nelson LD, Crowder E et al: Mutations in exon 17B of cartilage oligomeric matrix protein (COMP) cause pseudoachondroplasia. Nat Genet 1995; 10: 325-329.

30 Delot E, Brodie SG, King LM, Wilcox WR, Cohn DH: Physiological and pathological secretion of cartilage oligomeric matrix protein by cells in culture. J Biol Chem 1998; 273: 26692-26697.

31 Ikegawa S, Ohashi H, Nishimura G et al: Novel and recurrent COMP (cartilage oligomeric matrix protein) mutations in pseudoachondroplasia and multiple epiphyseal dysplasia. Hum Genet 1998; 103: 633-638.

32 Czarny-Ratajczak M, Lohiniva J, Rogala P et al: A Mutation in COL9A1 Causes multiple epiphyseal dysplasia: further evidence for locus heterogeneity. Am J Hum Genet 2001; 69: 5.

33 Jackson GC, Barker FS, Jakkula E et al: Missense mutations in the beta strands of the single A-domain of matrilin-3 result in multiple epiphyseal dysplasia. J Med Genet 2004; 41: 52-59.

34 Department of Health: Our inheritance, our future - realising the potential of genetics in the NHS; June 2003, ( www.dh. gov.uk/PolicyAndGuidance/HealthAndSocialCareTopics/Genetics/) accessed, September 2004 